Proceeding Series of the Brazilian Society of Computational and Applied Mathematics

\title{
Refining natural frequencies of linear second-order vibrating systems
}

\author{
João Batista da Paz Carvalho ${ }^{1}$ \\ Instituto de Matemática e Estatística, UFRGS, Porto Alegre, RS \\ Julio Cesar Claeyssen ${ }^{2}$ \\ Instituto de Matemática e Estatística, UFRGS, Porto Alegre, RS
}

Resumo. Natural frequencies of second order linear vibrating structures are frequently computed through reduction to generalized first order eigenvalue problem. We show how even complex second-order eigenvalues can be refined using Krylov-type strategies that apply recursion, shifts and inversion, using the original system matrices. Benchmarks with test matrices are presented.

Palavras-chave. second-order, natural-frequency, vibration, structure, refinement

\section{Introduction}

Second-order mechanical linear systems can be described, in the state-space form, by

$$
M \ddot{q}+C \dot{q}+K q=f,
$$

for symmetric matrices $M, C, K \in \mathbb{R}^{n \times n}$ called, respectively mass, damping and stiffness matrices. This is the case of vibrating structures such as bridges, buildings, transmission towers and others. $M$ is usually positive definite, while $K$ is usually positive semi-definite. If $C$ cannot be obtained, it is usually assumed the proportional damping hypothesis:

$$
C=\alpha M+\beta K, \alpha>0, \beta>0 .
$$

Important properties regarding stability and control of system (1) are determined by algebraic facts on the solutions pairs $\left(\lambda_{i}, x_{i}\right)$ of the second order eigenvalue problem

$$
\lambda^{2} M x+\lambda C x+K x=0 .
$$

which is usually transformed into a generalized first order eigenvalue problem

$$
\lambda B y=A y
$$

by defining $u=\lambda x, v=x, y=\left[\begin{array}{l}u \\ v\end{array}\right]$ and arranging the equations in a few possible ways:

\footnotetext{
${ }^{1}$ joao.carvalho@ufrgs.br

2julio@mat.ufrgs.br
} 
C1: keeping the symmetry in both sides:

$$
\lambda\left[\begin{array}{cc}
-M & 0 \\
0 & K
\end{array}\right]\left[\begin{array}{l}
u \\
v
\end{array}\right]=\left[\begin{array}{cc}
C & K \\
K & 0
\end{array}\right]\left[\begin{array}{l}
u \\
v
\end{array}\right]
$$

C2: keeping left side positive for $M$ and $K$ positive:

$$
\lambda\left[\begin{array}{cc}
M & C \\
0 & K
\end{array}\right]\left[\begin{array}{l}
u \\
v
\end{array}\right]=\left[\begin{array}{cc}
0 & -K \\
K & 0
\end{array}\right]\left[\begin{array}{l}
u \\
v
\end{array}\right]
$$

C3: keeping left side symmetric and positive for positive $M$ and $K$ :

$$
\lambda\left[\begin{array}{cc}
M & 0 \\
0 & K
\end{array}\right]\left[\begin{array}{l}
u \\
v
\end{array}\right]=\left[\begin{array}{cc}
-C & -K \\
K & 0
\end{array}\right]\left[\begin{array}{l}
u \\
v
\end{array}\right]
$$

C4: keeping left side positive for positive $M$ (useful if $K$ is singular):

$$
\lambda\left[\begin{array}{cc}
M & C \\
0 & M
\end{array}\right]\left[\begin{array}{l}
u \\
v
\end{array}\right]=\left[\begin{array}{cc}
0 & -K \\
M & 0
\end{array}\right]\left[\begin{array}{l}
u \\
v
\end{array}\right]
$$

After the reduction to (4) is established, if $B$ is positive definite, a very used approach to further simplification uses the Cholesky Factorization $L L^{T}=B$ which implies

$$
\lambda L L^{T} x=A x \Leftrightarrow \lambda L^{T} x=L^{-1} A L^{-T} L^{T} x \Leftrightarrow \bar{A} w=\lambda w, L^{T} x=w .
$$

This reduction to the first order eigenvalue problem, when $B$ is symmetric positive definite, is just one of the computational strategies available to solve (4). Other strategies for solving (4) include the QZ iteration [6], which is a generalization of the QR iteration method [6] for a pair of matrices $(A, B)$, through reduction to a Hessenberg-triangular form [11].

Computational strategies to solve (3) without transformation to first order problem are usually applied for large and sparse linear second-order systems, and include the wellknown Jacobi-Davidson method [8,12] and the SOAR method [1].

Well-known results on the sensitivity of problem (4) are given by several authors $[5$, 7,10] including the well-known Bauer-Fike theorem [6]. As for the original problem (3), only a few but insightful results exist, among them we refer to [9], which relates relative changes in second order eigenvalues and eigenvectors with relative changes in the matrices $M, C$ and $K$, in some matrix norm.

Since the matrices of problem (4) are obtained composing the matrices of problem (3) into double-sized matrices, and since no result on relationship between estimates for the two problems exist, one should expect to find situations in which (3) is far better conditioned than (4), possibly depending on which equation was used for the reduction.

A previous work [3] gathered computational evidences of this conjecture by benchmarking matrix datasets that showed that real eigenvalues obtained through reduction to (4) could yield to much better solutions of (3), obtained by using Krylov-subpace type techniques, real shifting, and by exploiting properties of the original system matrices. We propose here to extend those strategies, and to show how complex valued shifts and complex arithmetic strategies are able to refine any isolated solution of (3).

In section 2, we present our methodology and main definitions. In section 3, we present numerical results with public-domain test data comming from real-life vibrating structures. 


\section{Methods}

\subsection{Smallest singular value ratios}

Let $\left(\lambda_{i}, x_{i}\right), i=0, \ldots, 2 n-1$ be exact values of eigenpairs of (3) and let $\mu$ and $\mu^{*}$ be approximations that are close to $\lambda_{i}$. Since any matrix $W_{i}=\lambda_{i}^{2} M+\lambda_{i} C+K$ is singular, we will compare $\mu$ and $\mu^{*}$ on the grounds of how much singular they turn this matrix to be. Define the nullity ratio for a pair of approximations $\left(\mu, \mu^{*}\right)$ by

$$
N_{R}\left(\mu, \mu^{*}\right)=\frac{-\log _{10}\left(\min \left(\operatorname{svd}\left(\left(\mu^{*}\right)^{2} M+\mu^{*} C+K\right)\right)\right)}{-\log _{10}\left(\min \left(\operatorname{svd}\left(\mu^{2} M+\mu C+K\right)\right)\right)}
$$

where $\min (\operatorname{svd}(U)))$ stands for the smallest singular value of a matrix $U$. If $N_{R}\left(\mu, \mu^{*}\right)$ is greater than one, than $\left(\mu^{*}\right)$ approximates some $\lambda_{i}, i=1, \ldots, 2 n-1$, better than $\mu$.

Methodologically, $\mu$ is obtained through reduction to (4), while $\mu^{*}$ is obtained through a refinement technique that uses $\mu$ and the equation (3).

\subsection{The QZ method}

One of the most used computational strategies for solving (4) is the QZ method. The pair of matrices $(A, B)$ is reduced to a quasitriangular-triangular pair $(H, T)$ through orthogonal matrices $U$ and $V$ :

$$
U^{T} A V=H, U^{T} B V=T
$$

An iterative process, starting from matrices $H_{0}=H$ and $T_{0}=T$ and using QR decompositions, constructs a sequence of matrices $\left(H_{k}, T_{k}\right)$ that usually converges ${ }^{3}$ to the generalized Real Schur decomposition of $(A, B)$ from where real and complex eigenvalues can be obtained. More details can be found in [6].

\subsection{Second-order Arnoldi method}

This strategy [2] applies Fundamental solutions [4] to derive a recurrence

$$
\left\{\begin{array}{l}
M Y_{k+2}+C Y_{k+1}+K Y_{k}=0, \quad k=0,1,2,3, \ldots \\
Y_{0}=U_{0}, M Y_{1}=V_{0}
\end{array}\right.
$$

of matrices $\left\{Y_{k}\right\} \in \mathbb{R}^{n \times p}$ spanning Krylov-type like spaces from which approximations to dominant and isolated sets of eigenvectors can be computed. This strategy is closely related to the one in [1]. It is shown in [2] that, in order to construct a sequence $\left\{\alpha_{k}\right\}$ converging to a real single isolated eigenvalue $\lambda_{i}$ that is the closest to a given real number

\footnotetext{
${ }^{3}$ numerical analysis' strategies, like shifting, are applied to speed-up this convergence
} 
$\sigma$, one can shift and invert (12) in order to derive the recurrence formulas ${ }^{4}$

$$
\begin{array}{r}
K_{\sigma} y_{k+2}+C_{\sigma} y_{k+1}+M y_{k}=0 \\
\eta_{k}=\left|y_{k+1}\right|_{2}, y_{k+2} \leftarrow \frac{y_{k+2}}{\eta_{k}}, y_{k+1} \leftarrow \frac{y_{k+1}}{\eta_{k}}, y_{k} \leftarrow \frac{y_{k}}{\eta_{k}} \\
\alpha_{k}=\frac{1}{y_{k+1}^{T} y_{k+2}}+\sigma
\end{array}
$$

for any starting vectors $y_{0}, y_{1} \in \mathbb{R}^{n}$, and for parametric matrices

$$
C_{\sigma}=C+2 \sigma M, K_{\sigma}=K+\sigma C+\sigma^{2} M .
$$

We observe that he recurrence above can indeed be carried out for complex $\sigma$, if

$$
\alpha_{k}=\frac{1}{\overline{y k+1}^{T} y_{k+2}}+\sigma
$$

replaces (15) in computational environments that work with complex valued numbers. We remark that $\bar{z}$ stands for the complex conjugate of $z$.

If one wants to derive real valued formulas to accomplish the this recurrence in computational environments that do not work with complex valued numbers, we suggest to following approach: write $\sigma=\phi+\imath \psi$ for real $\phi$ and $\psi$ and observe that complex valued matrices $C_{\sigma}$ and $K_{\sigma}$ in (16) can be written

$$
C_{\sigma}=D_{\sigma}+\imath E_{\sigma}, K_{\sigma}=F_{\sigma}+\imath G_{\sigma}
$$

for real valued matrices $D_{\sigma}, E_{\sigma}, F_{\sigma}$ and $G_{\sigma}$ satisfying

$$
\begin{array}{r}
D_{\sigma}=C+2 \phi M, E_{\sigma}=2 \psi M \\
F_{\sigma}=\left(\phi^{2}-\psi^{2}\right) M+\phi C+K, G_{\sigma}=2 \phi \psi M+\psi C .
\end{array}
$$

If we also write $\bar{y}_{k+1}^{T} y_{k+2}=p_{k}+\imath q_{k}$ then (13)-(15) are equivalent to

$$
\begin{array}{r}
{\left[\begin{array}{rr}
F_{\sigma} & -G_{\sigma} \\
G_{\sigma} & F_{\sigma}
\end{array}\right]\left[\begin{array}{l}
u_{k+2} \\
v_{k+2}
\end{array}\right]=-\left[\begin{array}{rr}
D_{\sigma} & -E_{\sigma} \\
E_{\sigma} & D_{\sigma}
\end{array}\right]\left[\begin{array}{l}
u_{k+1} \\
v_{k+1}
\end{array}\right]-M\left[\begin{array}{l}
u_{k} \\
v_{k}
\end{array}\right]} \\
\eta_{k}=\sqrt{\left|u_{k+1}\right|_{2}^{2}+\left|v_{k+1}\right|_{2}^{2}}, u_{k+2} \leftarrow \frac{u_{k+2}}{\eta_{k}}, v_{k+2} \leftarrow \frac{v_{k+2}}{\eta_{k}} \\
u_{k+1} \leftarrow \frac{u_{k+1}}{\eta_{k}}, v_{k+1} \leftarrow \frac{v_{k+1}}{\eta_{k}}, u_{k} \leftarrow \frac{u_{k}}{\eta_{k}}, v_{k} \leftarrow \frac{v_{k}}{\eta_{k}} \\
p_{k} \leftarrow u_{k+1}^{T} u_{k+2}+v_{k+1}^{T} v_{k+2}, q_{k} \leftarrow u_{k+1}^{T} v_{k+2}-v_{k+1}^{T} u_{k+2} \\
\alpha_{k}=\frac{p_{k}}{p_{k}^{2}+q_{k}^{2}}, \beta_{k}=\frac{-q_{k}}{p_{k}^{2}+q_{k}^{2}}
\end{array}
$$

and $\left\{\alpha_{k}+\imath \beta_{k}\right\}$ converges to the eigenvalue $\lambda_{i}$ which the closest to given $\sigma=\phi+\imath \psi$.

Unfortunately in (21) symmetry and positivity properties of the working matrices are lost. Therefore, depending on computational aspects like size and sparsity of the system matrices, working with equations $(13),(14)$ and (17), that is, with complex valued matrices, can still be preferred because it can exploit symmetry, positiveness, sparsity and other properties the original system matrices might have.

\footnotetext{
${ }^{4}$ it is necessary to fix a small mistake in equation for $\alpha_{k}$, adding the missing back-shift term $\sigma$
} 


\section{Results}

\subsection{Transmission tower with proportional damping}

The data comes from the set BCSSTRUC1 of the Harwell-Boeing collection available in the MatrixMarket website [13]. It regards a 153 d.o.f. transmission tower assumed to have proportional damping with $\alpha=0.01, \beta=0.0003$. These parameters were chosen so that the model has eigenvalue pairs sufficiently apart from each other, and then refinement technique proposed in last section could be applied successfully. This model has 153 complex conjugate eigenvalue pairs. Only eigenvalues with positive imaginary parts were refined. The experiments were done in an Intel Dual Core Pentium G630 2.7GHz Desktop under Ubuntu Linux and software Matlab 2012b, which is able to carry out double complex arithmetic operations with almost no adjust at all to the real data algorithms from [2].

Figures 1 and 2 show that the eigenvalues could be successfully improved through iterative refinement, yielding to nullity ratios varying from 1.1 to 2.8 , regardless of the reduction formula that was used.
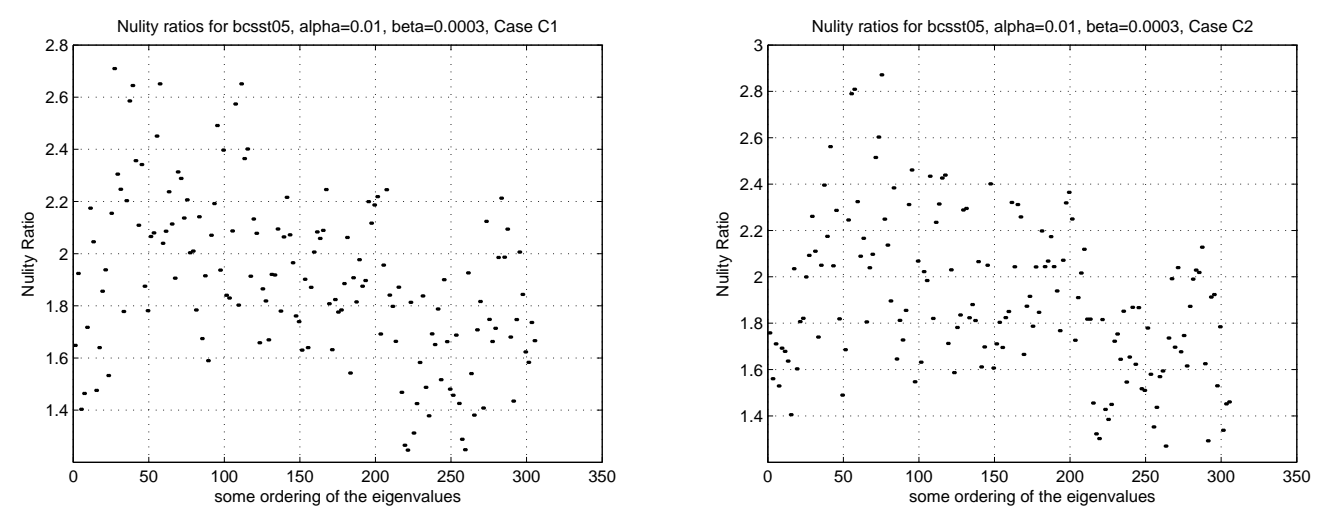

Figura 1: Nullity ratios for transmission tower, using formulas in (5) and (6).
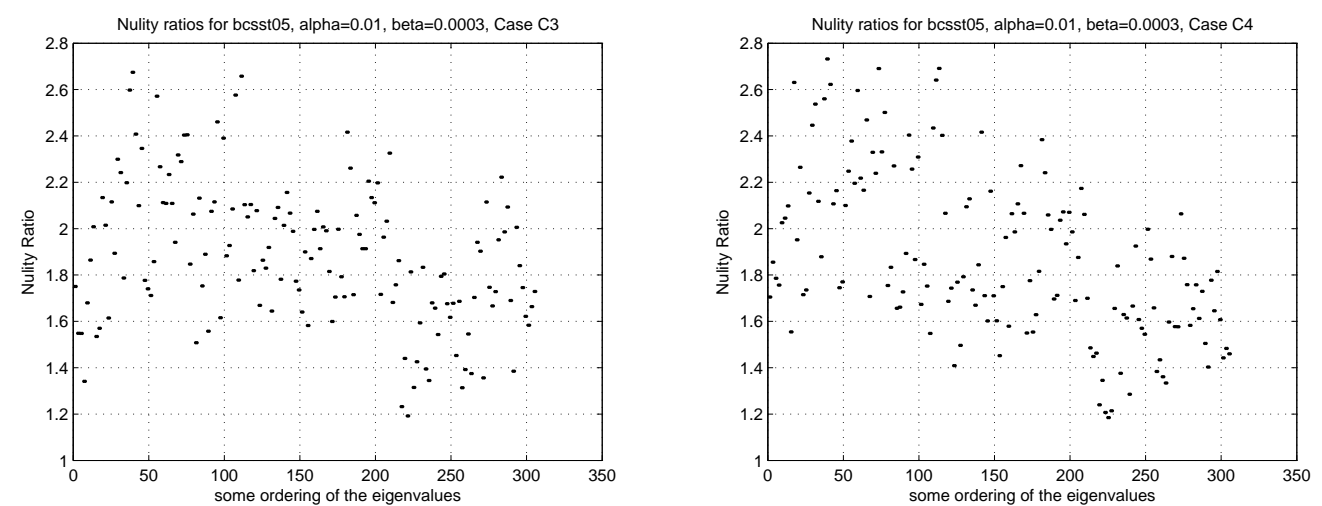

Figura 2: Nullity ratios for transmission tower, using formulas in (7) and (8). 


\subsection{Part of suspension bridge with proportional damping}

The data comes from the set BCSSTRUC3 of the Harwell-Boeing collection [13]. It regards a 817 d.o.f. part of a suspension bridge assumed to have proportional damping with the choices $\alpha=0.1, \beta=2.5 \cdot 10^{-5}$ so that all the 17 smallest magnitude eigenvalue pairs of the system are sufficiently isolated. The experiments were done in an Intel Dual Core Pentium G630 2.7GHz Desktop under Ubuntu Linux and Intel FORTRAN 90 compiler with double complex type data structures, with the aid of BLAS routines zaxpy, zspmv, dznrm2 and zscal, and LAPACK routines zsptrf and zsptrs.

Table 1 shows some outcomes in detail. It shows that the chosen subdominant set of frequencies was successfully refined with nullity ratios varying from 2 to 6.5. The singular-value computations for the nullity ratios were done in Matlab 2012b, however.

Tabela 1: Small subset of benchmark outcome for part of suspension bridge, using (6).

\begin{tabular}{|c|c|c|}
\hline$\mu$ & $\mu^{*}$ & $N_{R}$ \\
\hline$-0.051084766291962+9.317209566176491 i$ & $-0.051085162062924+9.317207486083117 i$ & 6.5 \\
$-0.050031035971404+1.582320118444923 i$ & $-0.050031327987364+1.582319772644305 i$ & 5.8 \\
$-0.050026089882555+1.442364218095966 i$ & $-0.050026036465881+1.442364263353126 i$ & 2.2 \\
$-0.050144725178335+3.400309779358339 i$ & $-0.050144557825537+3.400310511918943 i$ & 3.6 \\
$-0.050114336761382+3.027179214317865 i$ & $-0.050114579072032+3.027179263425846 i$ & 3.0 \\
$-0.050208054884942+4.082572936190894 i$ & $-0.050208373965475+4.082572271850640 i$ & 2.7 \\
$-0.050217871526307+4.173761937451974 i$ & $-0.050217785112027+4.173761748830215 i$ & 2.8 \\
$-0.050259834443233+4.564440771379331 i$ & $-0.050260458057345+4.564440653355708 i$ & 3.3 \\
$-0.050301883707487+4.917847147751918 i$ & $-0.050302346854857+4.917846893261517 i$ & 3.2 \\
$-0.050403489581206+5.685709562415424 i$ & $-0.050404122889866+5.685709332472713 i$ & 3.0 \\
$-0.050433043547391+5.886671744926964 i$ & $-0.050433193101629+5.886671778144693 i$ & 2.9 \\
$-0.050505086337247+6.357520894181837 i$ & $-0.050505257787513+6.357520917769455 i$ & 2.9 \\
$-0.050893272757203+8.455165630217271 i$ & $-0.050893655173541+8.455165505136536 i$ & 4.7 \\
$-0.050626496684400+7.078031137471627 i$ & $-0.050626263496229+7.078030564842803 i$ & 4.6 \\
$-0.050778545465319+7.891432941597847 i$ & $-0.050778465926318+7.891431787273492 i$ & 4.3 \\
$-0.050807677914873+8.039202592368438 i$ & $-0.050807891968324+8.039202449512876 i$ & 3.7 \\
\hline
\end{tabular}

\section{Conclusion}

Krylov-subspace type iterations using shifts and inversion, already shown to be able to refine real isolated eigenvalues of second order linear systems, were adapted to refine also complex conjugate isolated pairs. The proposed technique uses the original system matrices directly, exploiting properties like their symmetry and positiveness, and taking advantage that, in its original form, the eigenvalue problem seams to be far better conditioned than the generalized first order eigenvalue problem to which it is usually reduced to. Benchmarks using test data in computational environments capable to carry out complex valued arithmetic computations are made. Computational evidence has shown that a class of strategies that are usually applied in the context of large and sparse linear structures can also be of great benefit for improving the solution of the eigenvalue problem for small and medium sized dense structures, through iterative refinement. 


\section{Referências}

[1] Z. Bai and Y. Su, SOAR: A second-order Arnoldi method for the solution of the quadratic eigenvalue problem, SIAM J. Matrix Anal. Appl., vol. 26, n.3, 640-659 , (2005), DOI:10.1137/S0895479803438523.

[2] J. Carvalho and J. Claeyssen, Computation of extreme clustered natural frequencies of damped second-order linear systems, Proceedings of Conferência Brasileira de Dinâmica, Controle e Aplicações, vol. 1, 454-457, (2011).

[3] J. Carvalho and J. Claeyssen, Refining natural frequencies of damped linear vibrating structures, Proceedings of 34th Ibero-Latin American Congress on Computational Methods in Engineering, (2013).

[4] J. Claeyssen, On predicting the response of nonconservative linear vibrating systems by using dynamical matrix solutions, J. Sound and Vibration, vol. 140, 73-84, (1990).

[5] S. Eisenstat and I. C. Ipsen, Three absolute perturbation bounds for matrix eigenvalues imply relative bounds. SIAM J. on Matrix Analysis and Appl., vol. 20, n. 1, 149-158, (1998), DOI:10.1137/S0895479897323282.

[6] G. Golub and C. Van Loan. Matrix computations, Johns Hopkins University Press, Baltimore, MD, USA, (1996).

[7] D. Higham and N. Higham, Structured Backward Error and condition of generalized eigenvalue problems, SIAM J. Matrix Anal. Appl, vol. 20, n.2, 493-512, (1999), DOI:10.1137/S0895479896313188.

[8] G. Sleijpen and H. VanderVorst, A Jacobi-Davidson iteration method for linear eigenvalue problems, SIAM J. Matrix Anal. Appl., vol. 17, 401-425, (1996), DOI:10.1137/S0036144599363084.

[9] A. Sondipon. Rates of change of eigenvalues and eigenvectors in damped dynamic system, AIAA Journal, vol. 37, n.11, 1452-1458, (1999), DOI: 10.2514/2.622.

[10] G. W. Stewart. On the Sensitivity of the Eigenvalue Problem $A x=\lambda B x$. SIAM J. on Numerical Analysis vol. 9, n.4, 669-686, (1972), DOI:10.1137/0709056.

[11] A. Varga, Computation of irreducible generalized state-space realizations, Kybernetika, vol. 26, 89-106, (1990), DOI: 10.1.1.539.2629.

[12] Y. Zhou, Studies on Jacobi-Davidson, Rayleigh quotient iteration, inverse iteration generalized Davidson and Newton updates, Numerical Linear Algebra with Applications, vol.13, 621-642, (2006), DOI: 10.1002/nla.490.

[13] The Matrix Market directory on the web. Online reference and repository at http://math.nist.gov/MatrixMarket. 